\title{
DiabeTic RETINOPATHY ASSESSED BY DYNAMIC Light SCATTERING AND CORNEAL AUTOFLUORESCENCE
}

\author{
Luigi Rovati, ${ }^{\dagger}$ Franz Fankhauser II, ${ }^{\dagger, *}$ Franco Docchio, ${ }^{\dagger}$ and Jaap Van Best ${ }^{\dagger, *}$ \\ University of Brescia, Department of Electronics for the Automation, Via Branze 38 , \\ I-25123 Brescia; ${ }^{\ddagger}$ St. Thomas’s Medical and Dental School, Department of Ophthalmology Lambeth \\ Palace Road, SE1 7EH London; *Leiden University Medical Center, Department of \\ Ophthalmology J3-S Albinusdreef 2, 2333 ZA Leiden \\ (Paper JBO-167 received July 31, 1997; revised manuscript received Feb. 14, 1998; accepted for publication Mar. 2, 1998.)
}

\begin{abstract}
Autofluorescence of the cornea within specific wavelength region and dynamic light scattering measurements of ocular tissue have both been used for early-stage detection of the presence of diabetic retinopathy. In the present study, autofluorescence of the cornea and dynamic light scattering in the cornea and vitreous have been measured and compared using two innovative research instruments in twenty-two insulin-dependent diabetes mellitus patients, for the diagnosis of diabetic retinopathy. Corneal autofluorescence mean values in each diabetic retinopathy grade significantly correlated with dynamic light scattering measurements in the vitreous, and in the cornea, thus confirming that changes in the natural fluorescence is strictly correlated with molecular changes of ocular tissues. () 1998 Society of Photo-Optical Instrumentation Engineers. [S1083-3668(98)01103-4]
\end{abstract}

Keywords dynamic light scattering; corneal autofluorescence; ophthalmic instrument.

\section{INTRODUCTION}

It is known that diabetic mellitus (DM) is associated with a number of biochemical alterations in the eye as well as in other organs. Such alterations are glycosylation of macroproteins and other metabolic disorders. ${ }^{1-4}$ In general, the longer the DM duration, the more marked these alterations. In diabetics, an average prevalence of $52 \%$ of diabetic retinopathy (DR) has been reported. ${ }^{5}$ Diabetic alterations not only affect the retina: in early stages, alterations of the cornea, ${ }^{6,7}$ aqueous, ${ }^{8}$ lens, ${ }^{9,10}$ and vitreous $^{11,12}$ appear. In the vitreous, the threedimensional structure of the collagen fibers and the embedded hyaluronic acid molecules alter their tertiary structure in response to changes of their glycosylation and crosslinking. ${ }^{13}$ These changes at the molecular level may occur before any alteration in the retina can be observed by means of biomicroscopy. On the other hand, the vascular component of $\mathrm{DM}$ is interrelated to the metabolic disorder that also affects corneal tissues, resulting in an increase of the corneal autofluorescence (AF) emitted, among others, by the Schiff Base crosslinks of glycosylated macroproteins. In previous studies the relation between corneal autofluorescence ${ }^{14,15}$ and dy-

Address all correspondence to L. Rovati. Tel.: 39-30-37 15 447; Fax: 3930-38 0014; E-mail: rovati@bsing.ing.unibs.it namic light scattering (DLS) of ocular tissues ${ }^{16-18}$ have been investigated as a function of the grade of DR.

In the present study, corneal AF and the distribution of relaxation rate obtained by DLS measurements in the posterior vitreous and cornea were determined in insulin-dependent diabetes mellitus (IDDM) patients with three different grades of retinopathy, using systems specifically designed for possible use in-vivo as screening instruments. The two methods are compared in order to assess their usefulness as an indicator of the severity of DR.

\section{DESCRIPTION OF THE SYSTEMS}

\subsection{DYNAMIC LIGHT SCATTERING ANALYZER}

Dynamic light scattering is a well-known technique for the measurement of the random thermal movements of suspended particles, macromolecule solutions, and other complex liquid systems. ${ }^{19}$ The information achievable by DLS measurements is contained in the shape of the intensity autocorrelation function of the scattered light.

When the motion of the scattering centers is mainly Brownian, the autocorrelation function decreases monotonically to a final value ideally equal to one half of the initial value. A common way to extract information from the autocorrelation func- 
tion is via the analysis of its distribution of the relaxation rate. The distribution of the relaxation rate can be used to determine the protein size distribution in the volume under test (VUT) when the experiment is performed in vitro. Unfortunately, when the VUT is located in living eyes, the reduced freedom imposed by membranes and interactions between scattering centers may affect this interpretation. Therefore, the distribution of relaxation rate has been selected as a fingerprint of the ocular tissue under test. This approach, even if qualitative, has been proven to be efficient for diagnostic purposes. ${ }^{18}$

The human eye has the unique advantage of being transparent (to visible and near infrared radiation). Thus, several eye compartments with different kinds of macroproteins are accessible to examination by DLS. ${ }^{20}$

Structural alterations of the vitreous, associated with DM, can be detected by DLS. ${ }^{18}$ However, measurements are critical due to the limited scattering of these ocular tissue (the vitreous scatters roughly thirty times less than the lens), eye movements which are greatest at the anterior and posterior pole, and eye blinking. Moreover, the limited accessibility through the pupil as well as laser power safety considerations require the use of very high sensitivity DLS apparatus. Recently, with the help of high sensitivity instruments, first investigations of pathological process in the vitreous have been reported. ${ }^{21,22}$

In this experimental activity, DLS measurements on corneal and vitreal tissues have been performed with excitation laser beam power as small as 5-30 $\mu \mathrm{W}$. An outline of the measuring system is shown in Figure 1(a). The instrument consists of three main blocks: (i) the microscope extension, (ii) the control unit, and (iii) the digital correlator connected to a PC. The control unit and the microscope extension are connected by a pair of 3-m-long, single-mode optical fibers. The microscope extension is built into a standard ophthalmic microscope (mod. 900 BQ, Haag-Streit AG, Switzerland). Hollow excitation beam and single-mode fiber detector have been used to improve system performance, allowing reliable DLS measurements in the vitreous.

The hollow beam geometry allows optimal utilization of the pupil aperture for focusing of the excitation beam. ${ }^{23}$ The medical operator can observe the VUT directly and exploit the reflected annular beam at different interfaces to determine with sufficient precision the VUT position along the eye axis. For example, when the VUT is located in the middle of the cornea, the operator can see, by the binocular of the slit lamp, two identical rings backreflected by the corneal epithelium and endothelium; whereas, when the VUT is located in the vitreous, the ring diameter projected on the retina is inversely proportional to the distance from the retinal tissue.

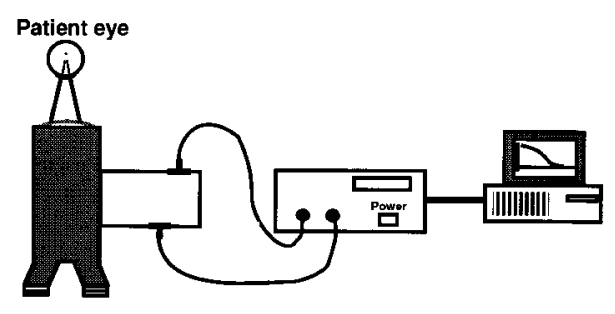

Standard Slit Lamp
(a) and mlcroscope extension Control Unit Personal Computer

Fig. 1 (a) Outline of the instrument for dynamic light scattering measurements. The instrument consists of three main blocks: (i) the microscope extension, (ii) the control unit, and (iii) the digital correlator connected to a PC. (b) Principle scheme of the instrument for corneal autofluorescence measurements. The excitation light from two blue LEDs passes through the excitation filters (460-480 nm). A suitable set of emission filters (500-540 nm) selects the wavelengths of interest, which are detected by the photomultiplier tube (PMT). The current signal at the output of the PMT is processed using custom front-end electronics.

A digital correlator (ALV-5000, ALV Lagen, Germany) is used for signal processing. This correlator employs the so called "symmetrical normalization scheme" and is, therefore, suitable for short averaging-time measurements. This fact can be exploited to reject artifacts due to eye blinking and movement averaging the data from five short measurement cycles during a period of $10 \mathrm{~s}$.

\subsection{CORNEAL FLUOROMETER}

Recently, corneal autofluorescence has been proposed as an ocular diagnostic tool for DR. ${ }^{14,15}$ The method is based on the sensible increase of the natural fluorescence of the corneal tissue within specific wavelength in the presence of the early stage of DR. The main advantages of this method are that corneal autofluorescence has been demonstrated to be unrelated to age and that the cornea is readily accessible for investigation.

The epithelium and the endothelium contains pyridine nucleotide and flavoproteins. The pyridine nucleotide shows fluorescence in the reduced state whereas flavoproteins show fluorescence in the oxidated form. The accumulation of these fluorophores in diabetic cornea related to the duration of diabetes cannot adequately explain the increased autofluorescence in patients with retinopathy. ${ }^{13}$ Two speculative explanations have so far been re- 
ported: (i) the vascular component of DM causing specific microangiopathy and consequently progressive retinopathy may induce a general metabolic disorder resulting in an increase of the corneal autofluorescence, or (ii) neovascularization mediating substances produced in the retina with retinopathy and inducing neovascularization of the iris may reach the cornea as well and consequently induce changes in the corneal metabolism resulting in increased value of $\mathrm{AF}^{14}$

In the present study, corneal AF has been measured by a dedicated research instrument ${ }^{24}$ using side illumination of the cornea by blue light emitting diodes (LED) light sources. Figure 1(b) shows the principle scheme of this instrument. The excitation light from two blue LEDs passes through a suitable set of barrier filters and impinges tangentially on the patients' cornea. The excitation light so obtained has a spectrum included between 460 and $480 \mathrm{~nm}$. The fluorescence light from the cornea is collected axially using a camera objective. A suitable set of emission filters select the wavelengths of interest (500-540 nm). The fluorescence light is then converted to an electric signal by a photomultiplier tube (PMT). The current signal at the output of the photomultiplier tube is processed using custom front-end electronics. The AF data are recorded in sixteen measurement cycles during a period of $10 \mathrm{~s}$ and the average value is considered.

\section{EXPERIMENTAL}

\subsection{SUBJECT SELECTION}

Twenty-two IDDM patients aged 16-73 years participated in this study, and were selected from the Eye Clinic of the University of Zürich. Great care was taken to select patients with normal aspect of all cornea layers and of the vitreous assessed by slit lamp examination. Individuals with contact lenses were excluded.

The modified Airlie House classification was used to grade the DR according to the following three grades:

Grade 1. No or negligible retinopathy; at most two microaneurysms per field.

Grade 2. Background or nonproliferative retinopathy; three or more microaneurysms per field, or one or more of the following items: retinal hemorrhages, hard or soft exudated and/or interetinal microvascular abnormalities (IRMA), venous beading.

Grade 3. (Pre-) proliferative retinopathy; hemorrhages and microaneurysms, and the following features present in more than two fields: soft exudates, IRMA, venous beading, and/or new vessel and fibrous proliferations, and/or vitreous hemorrhages.

Mean and standard deviation of the patients' age in the three groups are presented in Table 1.
Table 1 Mean and standard deviation of the patients' age in the three groups: grade 1: No or negligible retinopathy; grade 2: Background or nonproliferative retinopathy; grade 3: (Pre-) proliferative retinopathy.

\begin{tabular}{ccc}
\hline Group & $\begin{array}{c}\text { Number of } \\
\text { individuals }\end{array}$ & $\begin{array}{c}\text { Age (year) } \\
\text { (Mean } \pm \text { SD) }\end{array}$ \\
\hline Grade 1 & 4 & $52 \pm 16.70$ \\
Grade 2 & 4 & $73 \pm 4.35$ \\
Grade 3 & 14 & $56 \pm 16.20$ \\
\hline
\end{tabular}

\subsection{DATA ANALYSES}

Two DLS measurements in the posterior part of the vitreous and one in the cornea were performed in each eye using the instrument presented in Figure 1(a). The second measurement in the vitreous was used to establish that the first measurement had not been performed in spatial nonuniformity. Typical autocorrelation functions for in vitro measurement of a dilute sample of polystyrene latex spheres (nominal radius $91 \mathrm{~nm}$ ) and for in vivo measurements of corneal and in the vitreal tissue are shown in Figure 2. The amplitude of the dynamic part of the normalized autocorrelation function, known also as $\mathrm{S} / \mathrm{N}$ ratio of the autocorrelation function, approaches the theoretical limiting value of 1 for the in vitro measurement, whereas, for in vivo measurements in the cornea and in the vitreous, it is in the order of 0.8 and 0.4 , respectively. The reduction of this parameter for in vivo measurements with respect to the theoretical limit is mainly due to stray light from interfaces and static scattering centers. Nevertheless, these $\mathrm{S} / \mathrm{N}$ ratios are adequate for a correct data analysis.

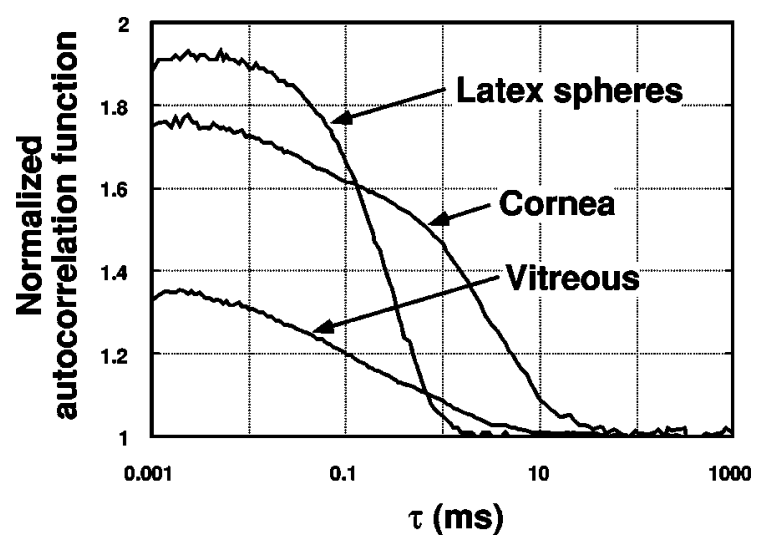

Fig. 2 Typical autocorrelation functions for dilute sample of polystyrene latex spheres (nominal radius $91 \mathrm{~nm}$ ) and for in vivo measurements in the cornea and in the vitreous. The amplitude of the dynamic part of the normalized autocorrelation function approaches the theoretical limiting value of 1 for the in vitro measurement, whereas, for in vivo measurements in the cornea and in the vitreous, it is in the order of 0.8 and 0.4 , respectively. 


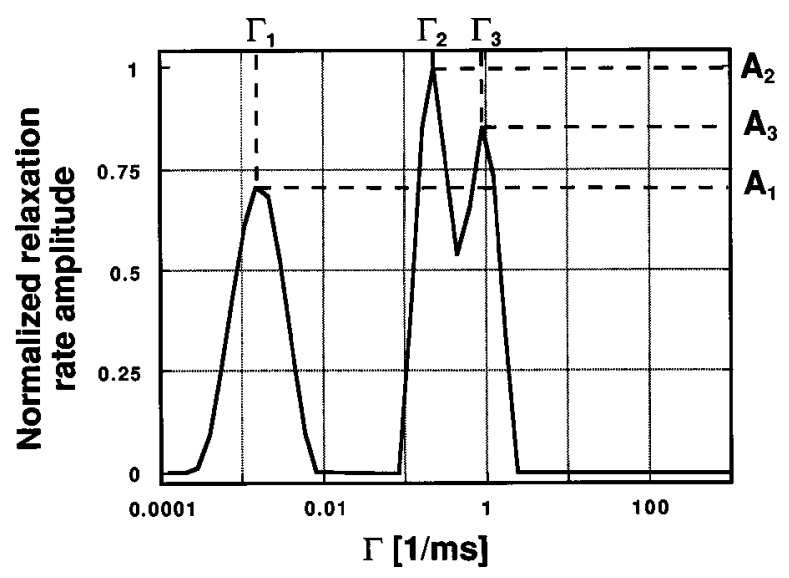

Fig. 3 Typical distribution of relaxation rate obtained performing the inverse Laplace transform of the autocorrelation function. The measurement has been performed in the cornea of a patient with retinopathy grade 1 .

The determined autocorrelation functions were analyzed using the program CONTIN (ALV Langen, Germany). This program performs the inverse Laplace transform of the autocorrelation function to obtain the distribution of relaxation rate. As shown in Figure 3, the typical spectrum exhibits a sequence of peaks with different amplitudes.

Two parameters were used to characterized the ocular tissues under test: (i) the abscissa Gam$\operatorname{ma}[\max$, measuring position] of the peak at the maximum amplitude and (ii) the center of gravity of the relaxation rate distribution, defined as:

$$
\text { Gamma[g, measuring position }]=\frac{\sum_{i=1}^{n} \Gamma_{i} \cdot A_{i}}{\sum_{i=1}^{n} A_{i}},
$$

where $\Gamma_{i}$ and $A_{i}$ are the abscissa and the amplitude of each peak, respectively (Figure 3 ). Average of the two measurements in the vitreous was calculated.

Three corneal autofluorescence measurements were performed in each eye, a few minutes after DLS examinations, using the ocular fluorometer. These measurements were performed in different corneal regions and the average value of the results were calculated. This operation minimizes the effect of spatial nonuniformity.
The two examinations were performed within a time interval of about $5 \mathrm{~min}$. Artifacts due to eye blinking and movement were minimized by averaging data from five measurement cycles of $2 \mathrm{~s}$ for DLS measurements and performing three AF measurements in each eye.

\subsection{RESULTS}

Mean and standard deviation of the DLS data and the corneal AF in each retinopathy grade are reported in Table 2. Experimental data was analyzed using the Mann-Whiter nonparametric test (Statistica, Statsoft Inc.). An average increase of about 70\% $(p=0.06)$ of the corneal AF between patients with negligible DR (grade 1) and with background DR (grade 2) was observed. Corneal AF mean value of patients with (pre-) proliferative DR (grade 3) decreases by about $26 \%(p=0.13)$ with respect to background DR (grade 2).

The Gamma[max, vitreous] mean value of patients with background DR (grade 2) were higher (about $45 \%, p=0.19$ ) when compared to mean values obtained with patients with both negligible (grade 1) or (pre-) proliferative DR (grade 3). Negligible dependence of the Gamma[max, cornea] values from the DR grade was observed $(p=0.8)$.

The Gamma[g, cornea] exhibited a significant dependence to the grade of DR. In patients with a negligible DR (grade 1), the Gamma[g, cornea] mean value was significantly higher (about 8.7 times, $p=0.0005$ ) than in patients with background DR (grade 2). Patients with (pre-) proliferative DR (grade 3) exhibit an intermediate value of Gam$\mathrm{ma}[\mathrm{g}$, cornea]. Negligible dependence of the Gam$\mathrm{ma}[g$, vitreous] from the $\mathrm{DR}$ was observed $(p=0.8)$.

The most significant parameters, i.e., mean values and standard errors of corneal AF, Gamma[max, vitreous], and Gamma[g, cornea], as a function of the DR grade are shown in Figures 4(a)-4(c).

In Figures 5(a)-5(c) the mean values of corneal AF, Gamma[max, vitreous], and Gamma[g, cornea] as a function of the patients' age are shown. Corneal AF means values in each DR grade correlated with Gamma[max, vitreous] and Gamma[g, cornea] as shown in Figures 6(a) and 6(b), respectively.

Table 2 Effect of diabetic retinopathy on corneal autofluorescence (AF) and dynamic light scattering results (Gamma[max, measuring position], Gamma[g, measuring position]). Gamma[max, measuring position] is the abscissa of the peak at the maximum amplitude of the relaxation rate distribution and Gamma[g, measuring position] is the center of gravity of the relaxation rate distribution.

\begin{tabular}{cccccc}
\hline Group & $\begin{array}{c}\text { Corneal AF (A.U.) } \\
\text { (Mean } \pm \text { SD) }\end{array}$ & $\begin{array}{c}\text { Gamma[max, vitreous] } \\
(1 / \mathrm{ms})(\text { Mean } \pm \text { SD) }\end{array}$ & $\begin{array}{c}\text { Gamma[max,cornea] } \\
(1 / \mathrm{ms})(\text { Mean } \pm \text { SD) }\end{array}$ & $\begin{array}{c}\text { Gamma[g,cornea] } \\
(1 / \mathrm{ms})(\text { Mean } \pm S D)\end{array}$ & $\begin{array}{c}\text { Gamma[g,vitreous] } \\
(1 / \mathrm{ms})(\text { Mean } \pm S D)\end{array}$ \\
\hline Grade 1 & $0.95 \pm 0.650$ & $0.406 \pm 0.330$ & $0.381 \pm 0.258$ & $2.93 \pm 2.380$ & $3.69 \pm 2.740$ \\
Grade 2 & $1.61 \pm 0.740$ & $0.599 \pm 0.280$ & $0.383 \pm 0.385$ & $0.30 \pm 0.148$ & $4.06 \pm 2.430$ \\
Grade 3 & $1.19 \pm 0.640$ & $0.462 \pm 0.220$ & $0.380 \pm 0.213$ & $1.10 \pm 1.220$ & $4.79 \pm 3.530$ \\
\hline
\end{tabular}




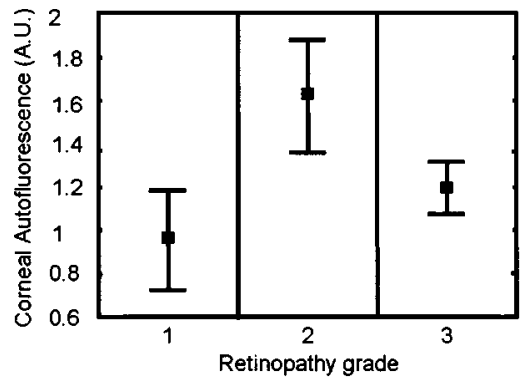

a)

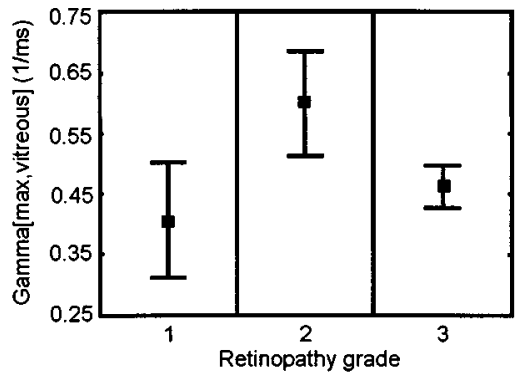

b)

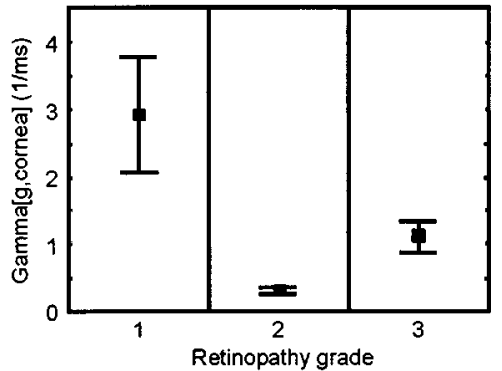

c)

Fig. 4 (a) Corneal autofluorescence expressed in arbitrary units, (b) Gamma[max, vitreous] values expressed in 1/ms, and (c) Gamma[g, cornea] values expressed in $1 / \mathrm{ms}$. Retinopathy is graded using the modified Airlie House classification (see the text). The closed squares and bars represent the mean value and the standard error of the mean in each group.

\subsection{DISCUSSION}

The present study demonstrates that both DLS in ocular tissue and corneal AF could be efficiently used for an early diagnosis of DR. High sensitivity and specificity could be obtained from corneal AF and Gamma[ $g$, cornea] by fixing a proper threshold to discriminate negligible (grade 1) to background DR (grade 2). These two parameters are not suitable to classify DR grade, since (pre-) proliferative DR (grade 3) could easily be confused with negligible DR (grade 1). Metabolic changes due to DR were observed using both DLS in the corneal tissue and corneal AF. The increase of the corneal AF between patients with negligible DR (grade 1) and with background DR (grade 2) was associated with a marked decrease in the mean value of Gamma $[g$, cornea]. This could be due to a metabolic disorder that results in an increase of the mean radius of the scattering molecules. However, it could be also related to a decrease of the molecular mobility, due for example to an increase of the local viscosity, or to interactions between scattering centres. Note that the decrease in the corneal AF between patients with background DR (grade 2) and with (pre-) proliferative DR (grade 3 ) is associated with an in- crease of Gamma[g, cornea] in the cornea. The high correlation between corneal AF means values and Gamma[g, cornea] [Figure 6(a)] demonstrates that changes in AF are induced by a structural change in the corneal tissue. No significant correlation with patients' age was observed for corneal AF, Gamma[max, vitreous], and Gamma[g, cornea].

Glycosylation of the hyaluronate molecules as well as the presence of pathologic plasma proteins, as may occur with a partial breakdown of the blood-retinal barrier (BRB) during the course of $\mathrm{DM}$, alters the tertiary and quaternary structure of the vitreous molecules as well as their diffusivity. Such changes are known from other tissues. ${ }^{25}$ In the vitreous they can be detected by DLS at a time when there are no visible alterations and no onset of DR. Because the breakdown of BRB causes significant changes of protein concentration in the vitreous first and then alterations of the corneal tissue, DLS in the vitreous should be more effective for an early detection of DR. It may be assumed that alterations due to DR manifest in the vitreous before the cornea due to the close spatial location of the vitreous and the retina. The increase of the Gam$\operatorname{ma}[\max$, vitreous] between patients with negligible

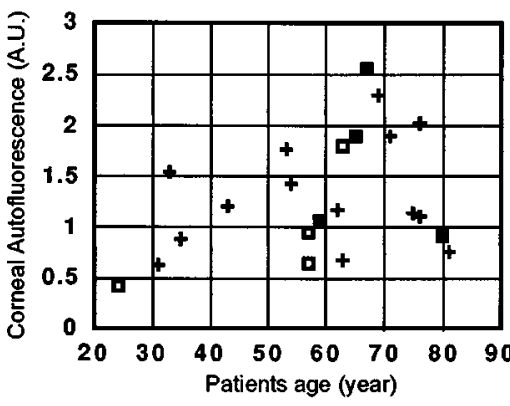

a)

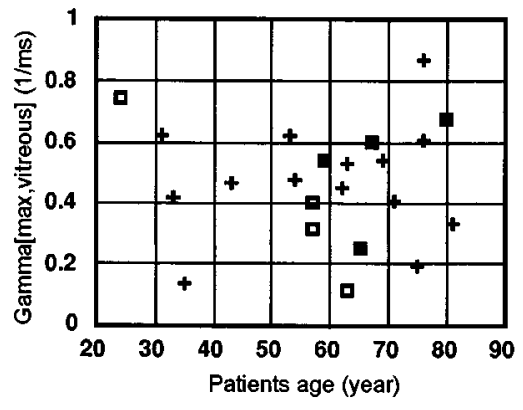

b)

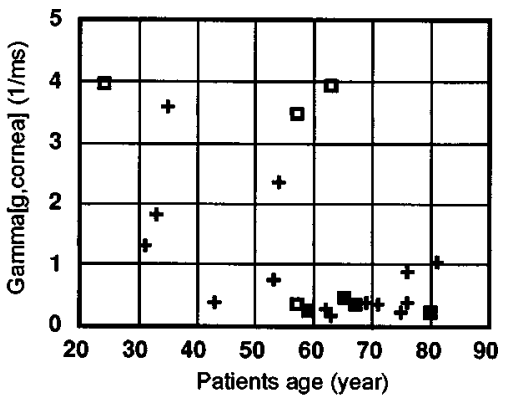

c)

Fig. 5 (a) Corneal autofluorescence, (b) Gamma[max, vitreous] values, and (c) Gamma[g, cornea] values as a function of the patients' age. Open squares: patients with retinopathy grade 1, i.e., no or negligible retinopathy. Closed squares: patients with retinopathy grade 2 , i.e., background or nonproliferative retinopathy. Crosses: patients with retinopathy grade 3, i.e., (pre-) proliferative retinopathy. 


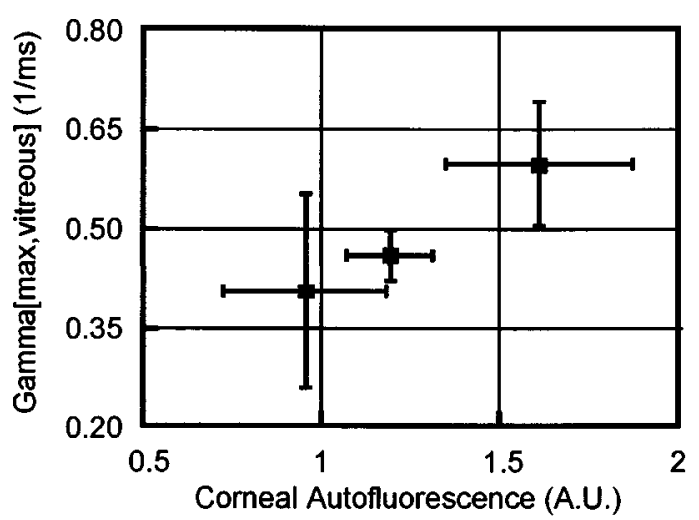

a)

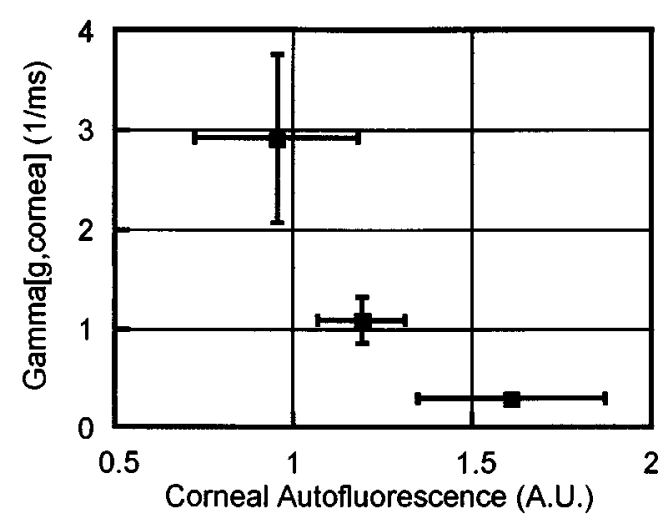

b)

Fig. 6 (a) Gamma[max, vitreous] and (b) Gamma[g, cornea] vs corneal autofluorescence (AF) in each DR grade. The closed squares and bars represent the mean value and the standard error of the mean in each group.

DR (grade 1) and with background DR (grade 2) could be due to alteration of the tertiary and quaternary structure of the vitreous molecules as well as their diffusivity. Even the mean value of Gamma[max, vitreous] significantly correlates with corneal AF [Figure 6(b)], confirming that the two phenomena are strictly connected. Nevertheless, corneal AF can be measured easily without burden to the patient. Theoretical advantages of vitreal measurements need to be investigated further under practical and clinical aspects.

\section{Acknowledgments}

The authors wish to thank Professor Gloor for the professional and logistic support at the University of Zürich-Augenklinik and all the kind volunteers who allowed the authors to examine them.

\section{REFERENCES}

1. Y. Maekawa, T. Nakamura, and R. Nogami, "Slerodermalike in a patient with insulin-dependent diabetes mellitus," J. Dermatol. 22(7), 508-511 (1995).

2. P. J. Beisswenger, Z. Makita, T. J. Curphey, L. L. Moore, S. Jean, T. Brinck-Johnsen, R. Bucala, and H. Vlassara, "Formation of immunochemical advanced glycosylation and products precedes and correlates with early manifestations of renal and retinal disease in diabetes," Diabetes 44(7), 824-829 (1995).

3. C. Sady, S. Khsrof, and R. Nagaraj, "Advanced Maillard reaction and crosslinking of corneal collagen in diabetes," Biochem. Biophys. Res. Commun. 214(3), 793-797 (1995).

4. M. Brownlee, "The pathological implications of protein glycation," Clin. Invest. Med. 18(4), 275-281 (1995).

5. F. A. L'Esperance, Jr. and W. A. James, Jr., "The problem of diabetic retinopathy," 11-20, in Diabetic Retinopathy, edited by H. L. Little, R. L. Jack, A. Patz, and P. H. Forsham, Thieme-stratton Inc., New York (1983).

6. L. I. Larsson, W. M. Bourne, J. M. Pach, and R. F. Brubaker, "Structure and function of the corneal epithelium in diabetes mellitus type I and type II," Arch. Ophthalmol. 114, 9-14 (1996).
7. J. Shimazaki, K. Tsubota, A. Yoshida, K. Tornheim, and R. A. Laing, "Change of corneal redox state in diabetic animal models," Cornea 14, 196-201 (1995).

8. F. Tost, P. Heilmann, C. Lautenschläger, "Aqueous flare measurement with a laser flare cellmeter in eyes with diabetic retinopathy," Ophthalmologica 209, 56-59 (1995).

9. J. Eppstein and S. E. Bursell, "Non-invasive detection of diabetes mellitus," Proc. SPIE 1641, 217-226 (1992).

10. B. E. Klein, R. Klein, Q. Wang, and S. E. Moss, “Older-onset diabetes and lens opacities. The beaver DAM eye study," Ophthalmic Epidemiol. 2, 49-55 (1995).

11. O. Lundquist and S. Oesterlin, "Glucose concentration in the vitreous of non-diabetic and diabetic human eyes," Graefes Arch. Clin. Exp. Ophthamol. 232, 71-74 (1994).

12. M. Bresgen, U. Baum, P. Esser, P. Wiedemann, and K. Heimann, "Die Proteinzusammensetzung des Glaskörpers bei proliferativer diabetischer Retinopathie," Ophthamologica 91, 758-762 (1994).

13. M. Brownlee, A. Creami, and H. Vlassara, "Advanced glycosylation and products in the tissue and the biochemical basis of diabetic complications," New Engl. J. Med. Suppl. 30, 1315-1321 (1988).

14. T. R. Stolwijk, J. A. van Best, J. A. Osterhuis, and W. Swart, "Corneal autofluorescence: An indicator of diabetic retinopathy," Invest. Ophthalmol. Vis. Sci. 33, $92-97$ (1992).

15. S. W. Chang, H. C. Hsu, F. R. Hu, and M. S. Chen, "Corneal autofluorescence and epithelial barrier function in diabetic patients," Ophthalmic Res. 27, 74-79 (1995).

16. R. Holden, G. A. Shun-Shin, and N. A. Brown, "Central corneal light scatter in long-term diabetics," Eye 8, 44-45 (1994).

17. U. Dürr, F. Fankhauser II, T. Walkow, L. Rovati, and J. Ricka, "In-vivo dynamic light scattering measurements in the cornea of human eye prior to and after excimer laser refractive surgery," Invest. Ophthalmol. Vis. Sci. 37, S568 (1996).

18. F. Fankhauser II, L. Rovati, J. Ricka, "In-vivo dynamic light scattering changes of the vitreous in diabetes mellitus," Invest. Ophthalmol. Vis. Sci. 37, S973 (1996).

19. Chu B, in Laser Light Scattering (Academic, New York, 1974).

20. G. B. Benedek, "Theory of the transparency of the eye," Appl. Opt. 10, 459-473 (1971).

21. L. Rovati, F. Fankhauser II, and J. Ricka, “Design and performance of a new ophthalmic instrument for dynamic light scattering in the human eye," Rev. Sci. Instrum. 67(7), 26152620 (1996).

22. R. R. Ansari and K. I. Suh, "Three-dimensional scanning of eye (lens and vitreous) using a newly developed dynamic 
light scattering probe," Proc. SPIE 2673, 12-20 (1996).

23. L. Rovati, "Hollow-beam geometry for dynamic light scattering measurements: a theoretical analysis," Appl. Opt. 36(34), 9083-9090 (1997).

24. J. A. van Best, F. Docchio, "A simple, low cost, portable corneal fluorometer for early detection of diabetic retinopathy," Appl. Opt. (submitted).

25. J. N. Lian, et al., "Change in the protein tertiary structure with non-enzymatic glycosylation of cal alfa crystalline," Biochem. Biophys. Res. Commun. 123, 899-906 (1984). 